\title{
Deep seafloor magnetic observations under GEOSTAR project
}

\author{
Angelo De Santis, Domenico Di Mauro, Lili Cafarella, Roberto D’Anna, Luis R. Gaya-Piqué, \\ Paolo Palangio, Giovanni Romeo and Roberta Tozzi \\ Istituto Nazionale di Geofisica e Vulcanologia, Roma, Italy
}

\begin{abstract}
Performing good quality magnetic observations is not an easy task; making them in the extreme marine environment is even more challenging. The European funded GEOSTAR project succeeded in reaching this difficult goal. After the shallow seawater test experiment performed in the Adriatic Sea in 1998, the main aims of the GEOSTAR project were achieved two years later during the six-month deep seafloor mission in the Tyrrhenian Sea at around $2 \mathrm{~km}$ depth. Details and results of the shallow seawater mission in the Adriatic Sea were published in previous articles. This paper is concerned with the deep seafloor mission in the Tyrrhenian Sea close to Ustica Island and presents some results related to the geomagnetic recordings.
\end{abstract}

Key words geomagnetic field - marine magnetometers - seafloor observations - skin depth

\section{Introduction}

The Earth's magnetism has its origin mainly within the fluid outer core by a dynamo mechanism that produces an essentially dipolar geomagnetic field (95\% of the total field), whose axis is slightly inclined with respect to the rotation axis by about $10^{\circ}$. In detail around $98 \%$ of the total field measured at the Earth's surface is of internal origin and $1 \%$ of the total field is due to crustal magnetisation characterising the different tectonic plates. The measured geomagnetic field also includes external fields whose sources are in the ionosphere and magnetosphere that, together with their crustal induced counterparts, contribute about $1 \%$ of the total field. Finally, the oceans also make a small

Mailing address: Dr. Angelo De Santis, Istituto Nazionale di Geofisica e Vulcanologia, Via di Vigna Murata 605, 00143 Roma, Italy; e-mail: desantisag@ingv.it contribution through the motions of seawater in the geomagnetic field. The geomagnetic field changes with time. Among its variations we recall, listed from longer to shorter characteristic periods: a) the secular variation, which is the slow (annual, decadal, and secular) trend of the field; b) solar diurnal variation, which is the daily oscillation due to the solar ionisation of the high atmosphere; c) bays, substorms and storms (range: from a few minutes to 1-2 days), the latter representing the most rapid variations with significant amplitude recorded by geomagnetic observatories on the Earth's surface.

Since land covers only around $1 / 3$ of the Earth's surface, to better understand the morphology of the geomagnetic field it is necessary to extend magnetic observations to the seafloors. Measuring the geomagnetic field beneath the sea has the characteristic that the external rapidly varying fields are partially screened by seawater (average conductivity of 3-4 S/m depending on salinity), while slowly varying fields, which are of internal origin, are practically unperturbed. The simultaneous magnetic variations recorded on the seafloor and at different locations on land are also related to the presence of electrical conductive layers or 
of lateral electrical discontinuities in the oceanic crust and upper mantle on a global scale and to the presence of local patterns in restricted areas.

The under-sea environment presents evident constraints on any kind of measurements. The pressure increases by 1 atm for every $10 \mathrm{~m}$ depth increment; hence at a depth of $2 \mathrm{~km}$ we have around $200 \mathrm{~atm}$. This high pressure requires that the instrumentation must be contained in strong, non-collapsible pressure cases. Corrosion is another adverse factor affecting many components of instruments or sensors, therefore proper protection must be adopted. The most important aspect for seafloor magnetic measurements is the seawater electrical conductivity. Salinity and pressure variations do not really influence this quantity significantly but temperature does. Seawater temperature decreases with depth: from $20-30^{\circ} \mathrm{C}$ (typical temperatures at middle temperate latitudes) at the surface to few degrees at the seafloor (e.g., around $3^{\circ} \mathrm{C}$ at $-3 \mathrm{~km}$ ) with very high stability. Correspondingly, the seawater electrical conductivity decreases with depth (from $6 \mathrm{~S} / \mathrm{m}$ at the surface to $3.5 \mathrm{~S} / \mathrm{m}$ at around $-1 \mathrm{~km}$ and to 3.3 at around $-2 \mathrm{~km}$ ). Below a depth of $2 \mathrm{~km}$ the conductivity has an almost constant value of $3.3 \mathrm{~S} / \mathrm{m}$; for the oceans this latter value can be considered as the average conductivity. Smaller seas such as the Mediterranean can present higher conductivity values that, in any case, are not higher than $4 \mathrm{~S} / \mathrm{m}$. The relatively high conductivity of seawater screens the propagation of electromagnetic (EM) variations. This can be easily understood by introducing the so-called skin depth $\delta$ (expressed in meters), of an EM variation of period $T$ in a medium of conductivity $\sigma$ as the propagation distance in a homogeneous conductor over which EM waves are attenuated by a factor of $\mathrm{e}^{-1}$, i.e.:

$$
\delta=503(T / \sigma)^{1 / 2}
$$

with $T$ in seconds and $\sigma$ in $\mathrm{S} / \mathrm{m}$ (Filloux, 1987). From the Fourier power spectrum of natural magnetic variations in open oceans, it can be deduced that there is no significant attenuation for periods longer than $30 \mathrm{~min}$. At periods shorter than $20 \mathrm{~min}$, seafloor magnetic varia- tions undergo considerable attenuation. If one needs a very high accuracy in studying magnetic variations between 1 day and $1 \mathrm{~h}$, water motions must be taken into account. However for many general purposes they can be neglected since they are one or two orders of magnitude smaller than natural variations; otherwise they should be monitored and then removed.

In summer 1998, the benthic station of the European Project GEOSTAR (GEophysical and Oceanographic STation for Abyssal Research; a project in the framework of the MArine Science and Technology Program, MAST III) was deployed for the first time in the Adriatic Sea, running for around 1 month and the magnetometers provided a good set of magnetic data (e.g., De Santis et al., 1999). The GEOSTAR deep-sea mission was then performed from September 25, 2000 till March 16, 2001 close to Ustica Island (38 $32^{\prime} 24^{\prime \prime} \mathrm{N}, 12^{\circ} 46^{\prime} 30^{\prime \prime} \mathrm{E}$; Sicily, Italy).

Details on the GEOSTAR project and its deployment and observational system can be found in some recent literature (e.g., Beranzoli et al., 1998, 2000, 2003; Gasparoni et al., 1998, 2002; Marvaldi et al., 1998, 2002; De Santis et al., 1999; Favali et al., 2002), therefore here we present only a brief description of the magnetic experiment of the deep-sea mission and some preliminary analysis of the magnetic data.

The next section introduces the aims of the project and some information about magnetic measurements within the GEOSTAR project. The next section is dedicated to the calibration and estimation of the orientation of the magnetometers. Two sections then describe the magnetic data together with some spectral analysis; another section shows some results obtained from the method of geomagnetic deep sounding. Finally the conclusions assess the most important results and findings.

\section{Seafloor magnetic measurements within GEOSTAR}

In terms of the magnetic experiment, the GEOSTAR project had the following objectives:

- Contribution towards achieving an even distribution of observatories around the world in order to improve the reliability of global 
models (such as the International Geomagnetic Reference Field, IGRF), and regional (Mediterranean) models of the magnetic field.

- Study of geomagnetic temporal variations: a) long period (e.g., secular variation); b) short period (studies of solar diurnal variation, geomagnetic storms and substorms at mid latitudes). Concerning long period variations, particular attention should be paid to the study of some peculiar aspects of the secular variation such as, for instance, the so-called jerks. Jerks are sudden changes in the rate of the secular variation that occur on a time scale of 1-2 years (e.g. Courtillot and Le Mouël, 1984). Although they are among the most investigated subjects of geomagnetism, their origin and their nature are still a matter of debate: seafloor magnetic field measurements might contribute to the elucidation of this intriguing phenomenon when compared with simultaneous ground data.

- Study of Earth's conductivity structure: contemporary magnetovariational investigations on the seafloor and at the surface allow, in principle, the detection of conductive layers in the oceanic crust and upper mantle; this kind of information is very important since electrical conductivity is closely related to temperature, phase and composition of materials.
- Coincidence with Oersted (1999-Present) and CHAMP (2000-present) satellite missions. Since the study of the radial variation of the magnetic field has always been one of the most interesting aspects of geomagnetism, it is very relevant to have contemporary magnetic measurements taken at three such different altitudes, i.e. from 700 to $300 \mathrm{~km}$ (satellite altitude), around $0 \mathrm{~km}$ (i.e. at the Earth's surface), a few $\mathrm{km}$ under the sea level (seafloor observations).

Two magnetometers were installed on the GEOSTAR benthic station for the Tyrrhenian deep-sea mission: a scalar Overhauser GSM19L proton magnetometer by GEM System Inc. (Canada) and a three-axis suspended magnetometer, designed and built in the Laboratories of Istituto Nazionale di Geofisica e Vulcanologia (Rome and L'Aquila, Italy). Both sensors were protected from high pressure by two suitable benthospheres.

The omnidirectional sensor of the scalar magnetometer provided the total intensity of the geomagnetic field with a nominal resolution of $0.1 \mathrm{nT}$, an absolute accuracy of $1 \mathrm{nT}$ and a power consumption of $1 \mathrm{~W}$ at a sampling rate of 1 value per minute.

The fluxgate three-component sensor was suspended to ensure its verticality. Its resolu-

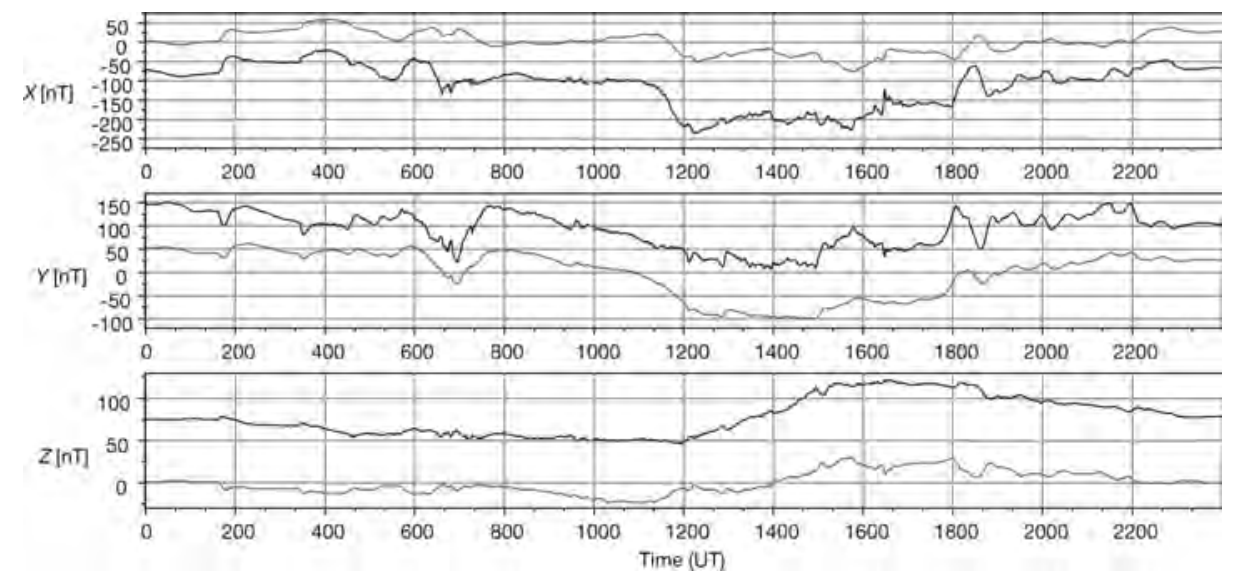

Fig. 1. October 5, 2000 - L'Aquila geomagnetic observatory (thick line) and GEOSTAR (thin line) $X, Y, Z$ components. $X, Y, Z$ are north, east and vertically downward magnetic components, respectively. An appropriate baseline must be added to each component. 


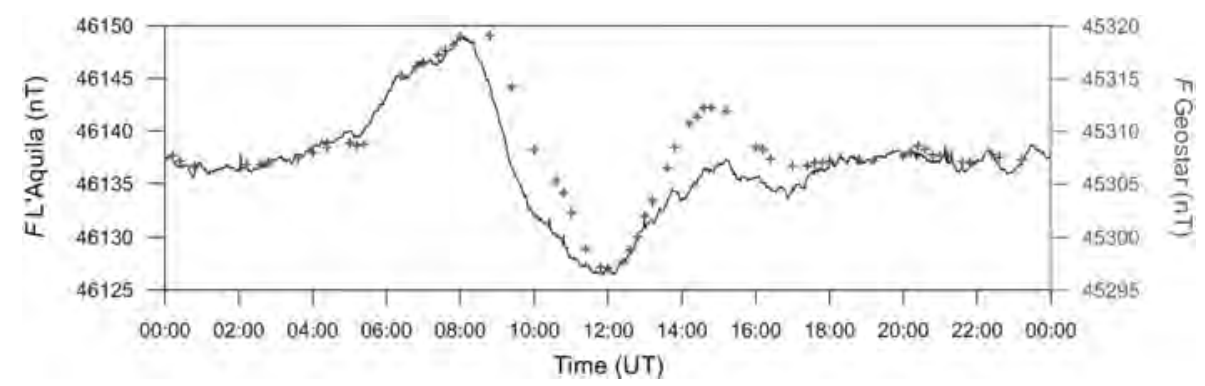

Fig. 2. March 15, 2001 - L'Aquila geomagnetic observatory (continuous line) and GEOSTAR (crosses) total field intensity, $F$.

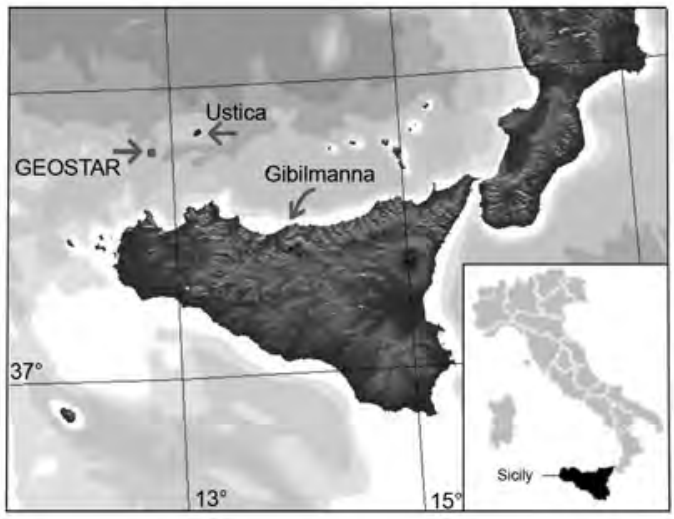

Fig. 3. Location of GEOSTAR site, Ustica and Gibilmanna (Sicily, Italy).

tion was $0.1 \mathrm{nT}$, with an absolute accuracy of 5$10 \mathrm{nT}$ and a power consumption of $2 \mathrm{~W}$ at a sampling rate of 6 values per minute. Its analog outputs were digitised by a 16 bits A/D converter, achieving a quantisation step size of $1.4 \mathrm{nT}$ in order to cover all possible ranges of the geomagnetic field.

The GEOSTAR deep-sea mission of 20002001 provided $4123 \mathrm{~h}$ of geomagnetic data. A sudden failure in the electronic device of the scalar magnetometer occurred at the very beginning of the mission and caused a reduction of the planned sampling rate of 1 value per minute and the achievement of a rate of only 1 value every twelve minutes.

In figs. 1 and 2 we show a comparison between GEOSTAR and L'Aquila observatory
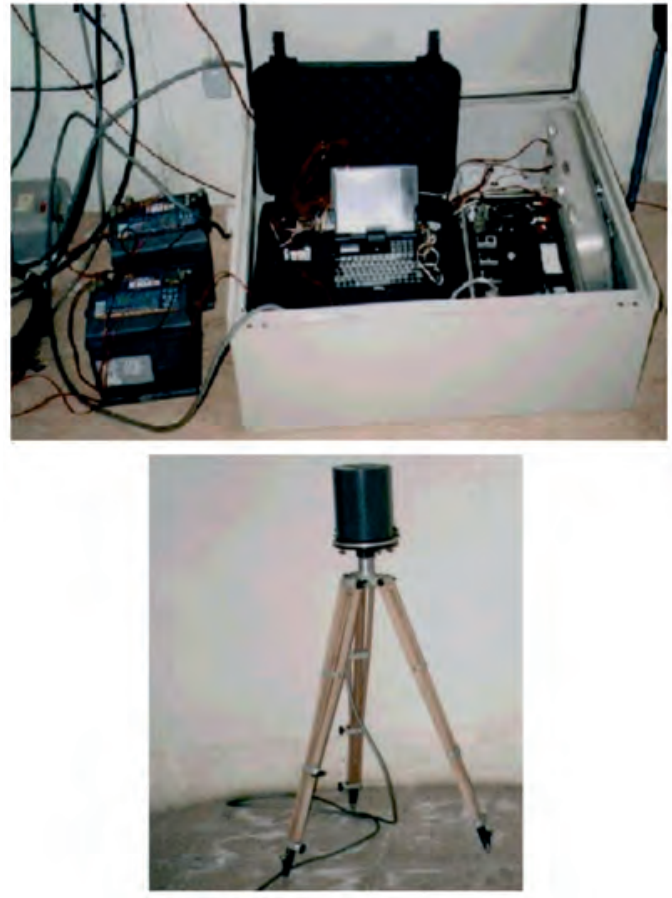

Fig. 4. Electronic equipment (top) and the three-axis fluxgate sensor (bottom) for the land stations.

$\left(42^{\circ} 23^{\prime} \mathrm{N}, 13^{\circ} 19^{\prime} \mathrm{E}, 0.7 \mathrm{~km}\right.$ altitude) vector magnetic components and total intensity of the magnetic field for October 5, 2000 (characterised by moderate magnetic activity) and March 15, 2001, respectively.

In order to have a reference station closer than L'Aquila and in close proximity to the GEO- 
STAR site, a land station was installed at Ustica Island $\left(38^{\circ} 42^{\prime} \mathrm{N}, 13^{\circ} 11^{\prime} \mathrm{E}, 0.2 \mathrm{~km}\right.$ altitude) and another at Gibilmanna $\left(37^{\circ} 59^{\prime} \mathrm{N}, 1^{\circ} 01^{\prime} \mathrm{E}, 1 \mathrm{~km}\right.$ altitude) (see fig. 3). Each station was based on a vector fluxgate sensor, a control unit and a data logger (fig. 4), using a solar panel for power supply. Moreover, at the beginning and at the end of the mission, absolute (static) measurements of the magnetic declination and inclination at Gibilmanna and at Ustica Island were performed. Such measurements were necessary to calibrate and validate the recordings made by the variographs.

\section{Calibration and orientation of GEOSTAR frame}

Two important considerations must be taken into account when analysing the GEOSTAR dataset: firstly the calibration and compensation for magnetic materials in the other instruments, and in the supporting structure of the module; secondly the orientation offset of the structure during the mission with respect to the true magnetic north.

For convenience, a dedicated calibration was carried out in the Italian magnetic observatory of L'Aquila (Central Italy) some days after the recovery of the benthic station (fig. 5). A comparison between the simultaneous recordings of the magnetic field components from L'Aquila and from the magnetometers mounted at the end of the booms on the submarine module, gives a quantification of the artificial offset due to GEOSTAR.

The compensation model used can be expressed as follows:

$$
B_{\text {ref }}=B_{\text {geos }}-B_{\text {perm }}-\delta \cdot B_{\text {geos }}
$$

where $B_{\text {ref }}$ is the magnetic field as recorded by the reference observatory, $B_{\text {geos }}$ is the magnetic field as observed by GEOSTAR, $B_{\text {perm }}$ is the permanent field due to the station frame's metal parts, and $\delta$ is a $3 \times 3$ matrix of constant coefficients characterising the induced field (seen by GEOSTAR). $\delta$ and $B_{\text {perm }}$ are unknowns with nine and three parameters, respectively. For instance, for the $x$ direction, we have

$$
\begin{aligned}
& B x_{\mathrm{ref}}=B x_{\mathrm{geos}}-B x_{\mathrm{perm}}-\left[\delta_{x x} \cdot B x_{\mathrm{geos}}+\delta_{x y} \cdot B y_{\text {geos }}+\right. \\
& \left.+\delta_{x z} \cdot B z_{\text {geos }}\right]
\end{aligned}
$$

where $B x, B y$ and $B z$ are the Cartesian components of the geomagnetic field in the different coordinate references.

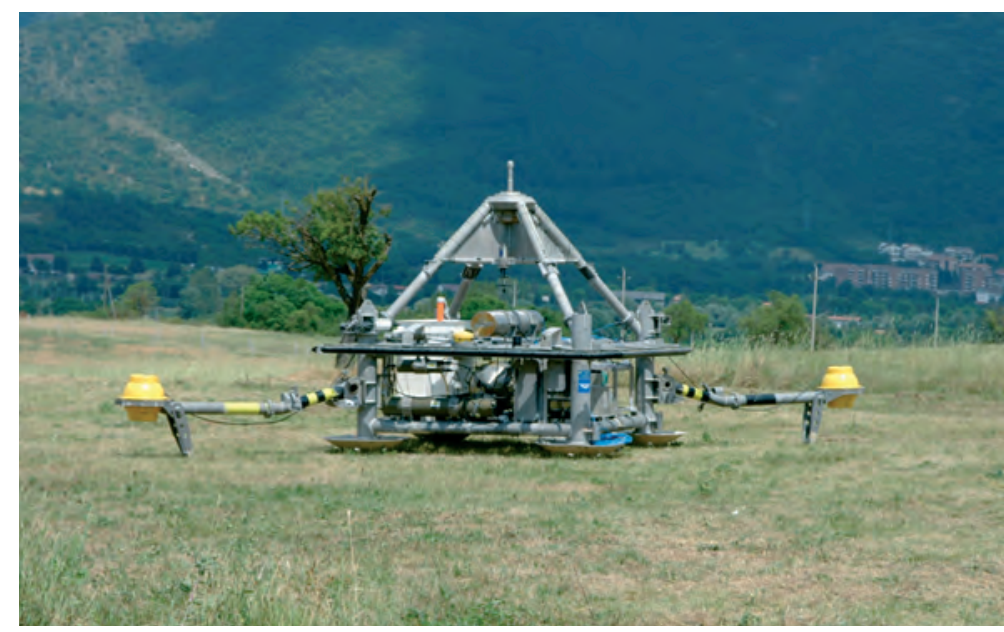

Fig. 5. GEOSTAR submarine module (frame) at L'Aquila during calibration and comparison with magnetic data from observatory. Magnetometers are mounted at the extremity of the oppositely extended booms. 
First we noticed that the original $B y_{\text {geos }}$ and $B x_{\text {geos }}$ had to be exchanged; then, the sign of $B z_{\text {geos }}$ had to be reversed. From now on we will denote the Cartesian components observed by GEOSTAR as those obtained after these changes.

We see that eq. (3.1) has two separate contributions, the permanent and induced parts, respectively. Inverting eq. (3.1) for the parameters $\delta$ when considering different orientations of the station with respect with true north, we found that the induced part is practically negligible; for what concerns the permanent part, we estimated the following values: $B x_{\text {perm }}=1075.4 \mathrm{nT}$,

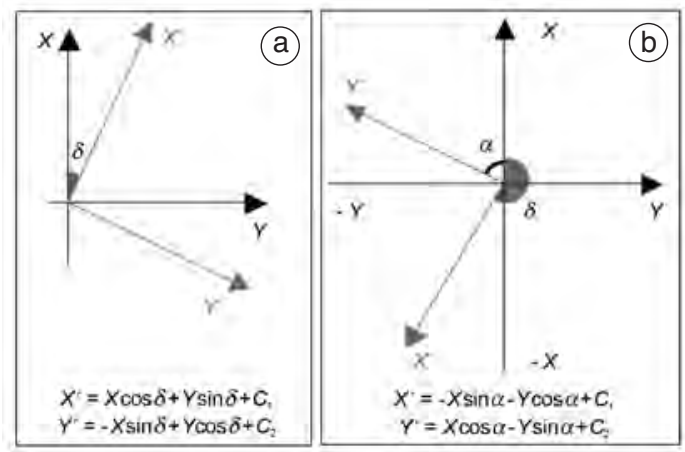

Fig. 6a,b. Two possible orientations of the GEOSTAR frame.
$B y_{\mathrm{perm}}=448.2 \mathrm{nT}$, and $B z_{\mathrm{perm}}=1077.6 \mathrm{nT}$. Apart from its intermittent difficulties, the scalar magnetometer provided values that need a correction of $-295 \mathrm{nT}$.

An estimated orientation of the whole station frame during the final release at a depth of $2000 \mathrm{~m}$ under the sea surface was known from the compass included in the 3D-ACM (threecomponent Acoustic Current Meter), which gave an angle of $+242^{\circ}$ (counter-clockwise from north). An in-depth evaluation of the GEOSTAR frame orientation was made comparing data recorded by GEOSTAR with data from the two land stations of Ustica Island and Gibilmanna and from L'Aquila Observatory. The comparison was made using least squares regression to discriminate between small or large angle of rotation, as shown in fig. 6a,b respectively.

To check the method we compared the horizontal $X, Y$ components as recorded at L'Aquila and Castello Tesino $\left(46^{\circ} 03^{\prime} \mathrm{N}, 11^{\circ} 39^{\prime} \mathrm{E}, 1.2\right.$ $\mathrm{km}$ altitude) observatories for a day of moderate magnetic activity (February 15, 2001) and then we calculated the rotation offset between L'Aquila Observatory-GEOSTAR and Ustica station-GEOSTAR. The compensation angle between the corresponding orthogonal sensor frames had a median value of $-1.9^{\circ}$, which is a small deviation, as expected for reference systems of observatories.

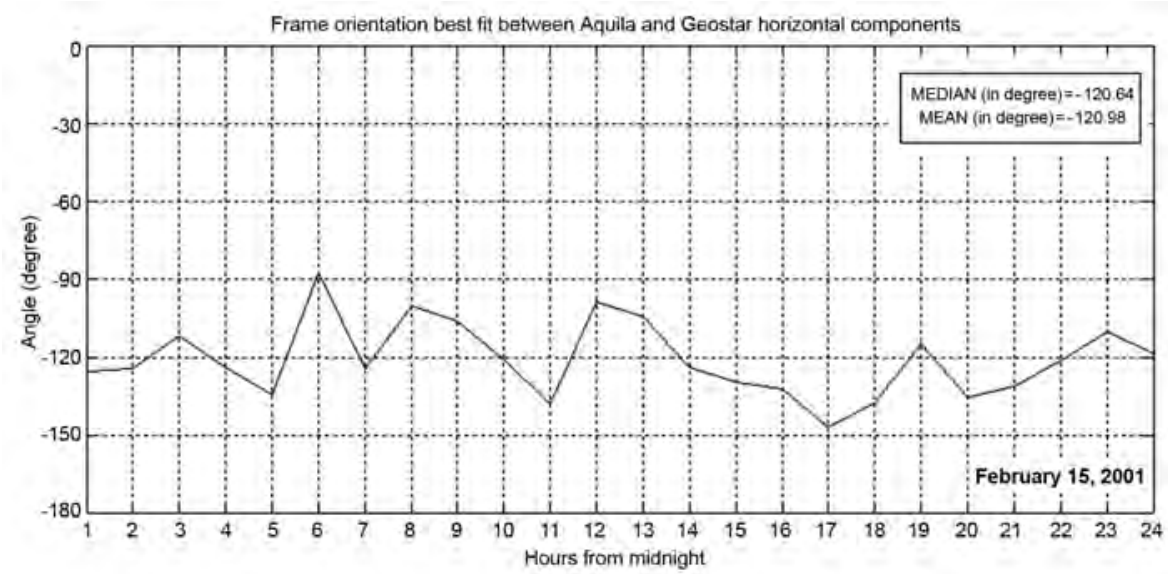

Fig. 7. Results of the orientation analysis between L'Aquila Observatory and GEOSTAR. 
For the same day we calculated the median (and the mean) values for the couples L'AquilaGEOSTAR (fig. 7) and Ustica-GEOSTAR, obtaining respectively $-120.64^{\circ}$ (mean $-120.98^{\circ}$ ) and $-119.97^{\circ}$ (mean $-123.73^{\circ}$ ) which confirm, within a few degrees, the values provided by the compass in the 3D-ACM.

\section{Data description}

Data validation for the whole GEOSTAR dataset was achieved by reducing any manipulation of the original recordings as much as possible. Each component was reconstructed applying the rotation and calibration corrections as described in the previous section to the vector magnetometer recordings. The six values per minute were averaged to produce 1-min values after applying a spike remover filter which acted on 6 to 15 points for each day. A list of all missing data segments (for both vector and scalar recordings) is given in table I.

The scalar magnetometer provided a total of 20615 available data. The leakage of data corresponds to the ratio of available data over the
Table I. Missing data segments of the GEOSTAR deep-sea mission.

\begin{tabular}{ccc}
\hline \hline Data & Starting time & Ending time \\
\hline 27/09/2000 & $13: 00$ & $13: 25$ \\
30/09/2000 & $13: 00$ & $14: 25$ \\
03/10/2000 & $03: 00$ & $04: 25$ \\
$08 / 10 / 2000$ & $12: 00$ & $13: 25$ \\
$09 / 10 / 2000$ & $08: 00$ & $08: 25$ \\
20/10/2000 & $20: 00$ & $21: 25$ \\
$26 / 10 / 2000$ & $05: 00$ & $05: 25$ \\
$04 / 11 / 2000$ & $07: 00$ & $07: 25$ \\
$16 / 11 / 2000$ & $02: 00$ & $03: 25$ \\
$02 / 12 / 2000$ & $00: 00$ & $01: 25$ \\
$02 / 12 / 2000$ & $20: 00$ & $20: 25$ \\
$03 / 12 / 2000$ & $00: 00$ & $00: 49$ \\
$20 / 12 / 2000$ & $22: 00$ & $22: 25$ \\
$06 / 01 / 2001$ & $01: 00$ & $01: 25$ \\
$25 / 01 / 2001$ & $16: 00$ & $16: 25$ \\
$28 / 01 / 2001$ & $16: 00$ & $16: 25$ \\
$22 / 02 / 2001$ & $09: 00$ & $10: 25$ \\
$26 / 02 / 2001$ & $05: 00$ & $05: 25$ \\
$28 / 02 / 2001$ & $13: 00$ & $13: 25$ \\
$03 / 03 / 2001$ & $01: 00$ & $02: 25$ \\
\hline
\end{tabular}

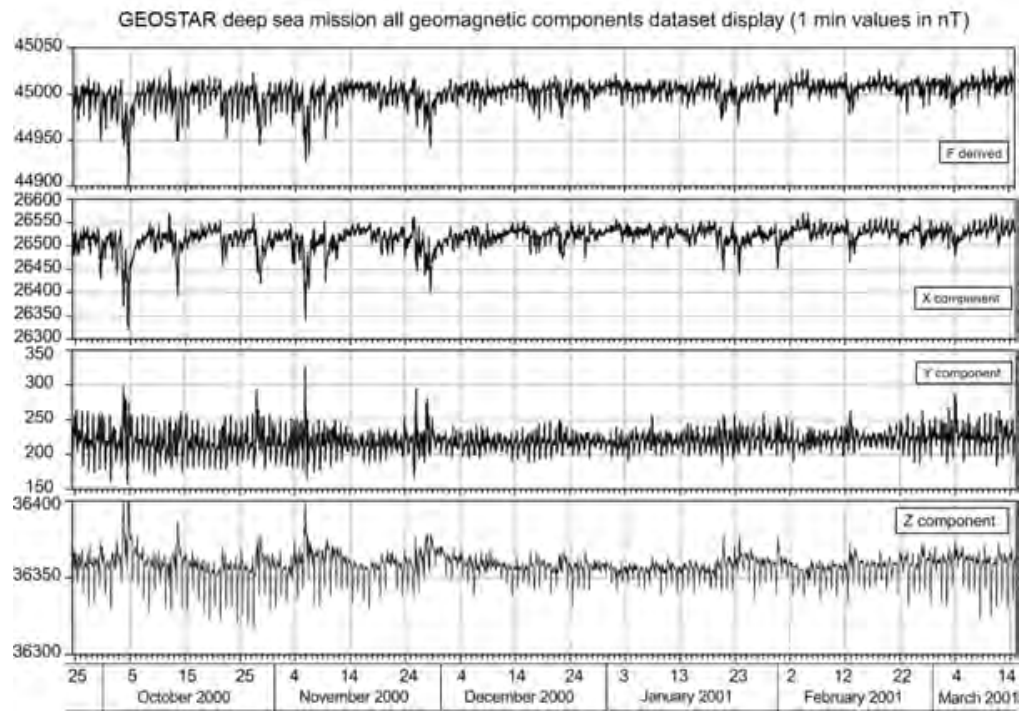

Fig. 8. Six months of seafloor recordings. Cartesian components and derived $F$ are displayed. Daily variations and some magnetically active periods are clear in all curves. 
Angelo De Santis et al.

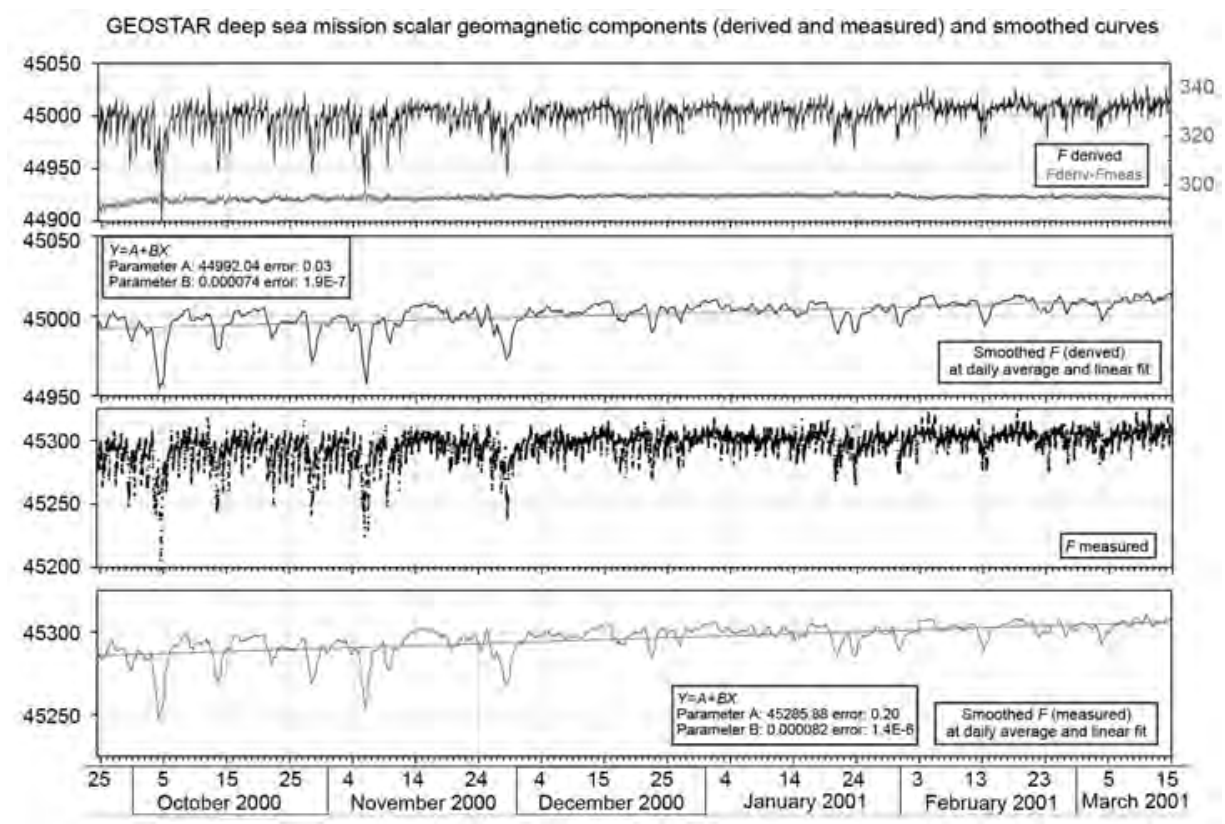

Fig. 9. Comparison between derived and measured $F$ and their difference. Parameters of linear fits on their smoothed curves are also reported.
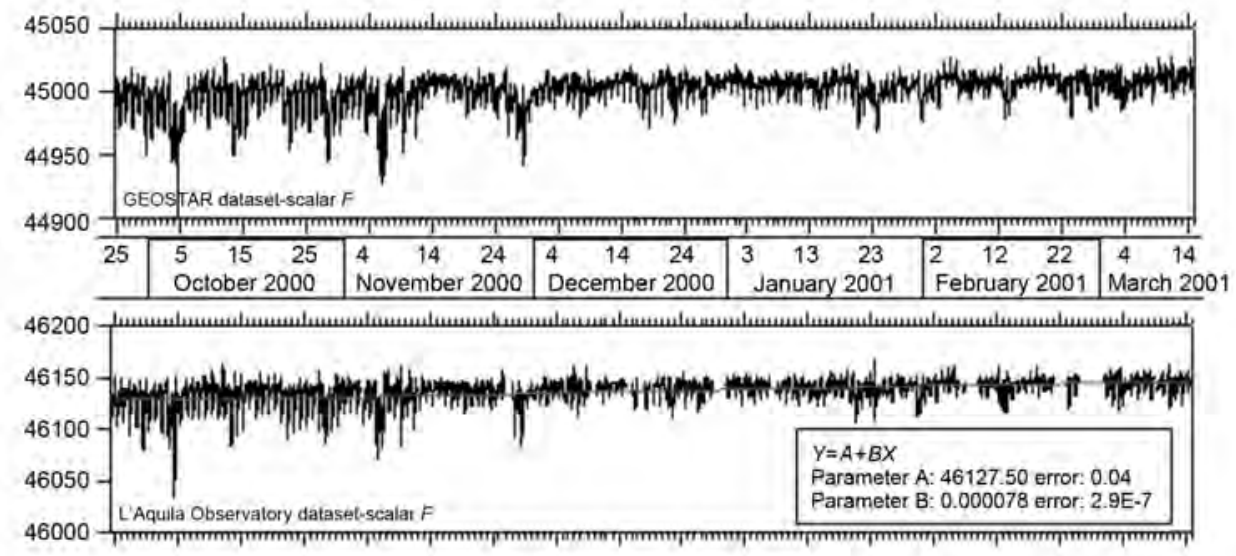

Fig. 10. Total geomagnetic field $F$ : comparison between GEOSTAR and L'Aquila Observatory. Dataset time windows: September 25, 2000 at 11:00 - March 16, 2001 at 00:59 - tick mark at 23:59 of the day.

total duration (in minutes) of the mission. For the scalar data we have about $91.6 \%$ of missing data. The vector magnetometer provided a total of 1484282 available data but between 6 and 15 spikes are present for each 24-h segment of recording. In this case we have about $0.2 \%$ of missing data. The total intensity $F$ of the magnetic field was then derived as the square root of the sum of the squares of the three re-oriented and calibrated components. 
The three geomagnetic components together with the $F$ values obtained in this way are displayed in fig. 8 . The total intensity directly measured by the scalar magnetometer mounted on GEOSTAR is displayed in fig. 9 (third horizontal panel) as single points, since the instrumentation collected only 1 data point every $12 \mathrm{~min}$.

The difference between derived and measured $F$ values is shown as scattered points in the lower part of the upper panel of fig. 9. From this panel an adjustment of the system in the first 10 days is clear, after which the value of difference is quite stable with an average value of 294.88 $\mathrm{nT}(s d=1.15 \mathrm{nT})$. A greater spread of points in the initial three months was due to strong magnetic activity occurring at that time. This reflects the instability conditions during strong magnetic field activities. Besides the good agreement between the derived and measured $F$ daily smoothed curves in fig. 9 , we observe that the daily variations completely vanish in the linear fit applied to the smoothed curves. This shows the good agreement between the six- month increases in the two datasets: that derived at the GEOSTAR site and that measured at L'Aquila Observatory (fig. 10) with a similar value of secular variation of around 42 nT/year.

\section{Data analysis}

In order to faithfully compare data recorded at L'Aquila Observatory with those recorded by GEOSTAR in terms of the energy and dynamics of the corresponding time series, we chose three days characterised by different geomagnetic activity and evaluated their power spectra.

The $K$ activity index computed from L'Aquila Observatory geomagnetic recordings allows us to make an appropriate selection of data segments to be used for both power spectra analysis and electromagnetic induction studies (fig. 11). For our purposes we considered the daily sum of the corresponding eight $K$-indices, calling this value the daily total $K$-index. Then we selected three days with different levels of magnetic activity.

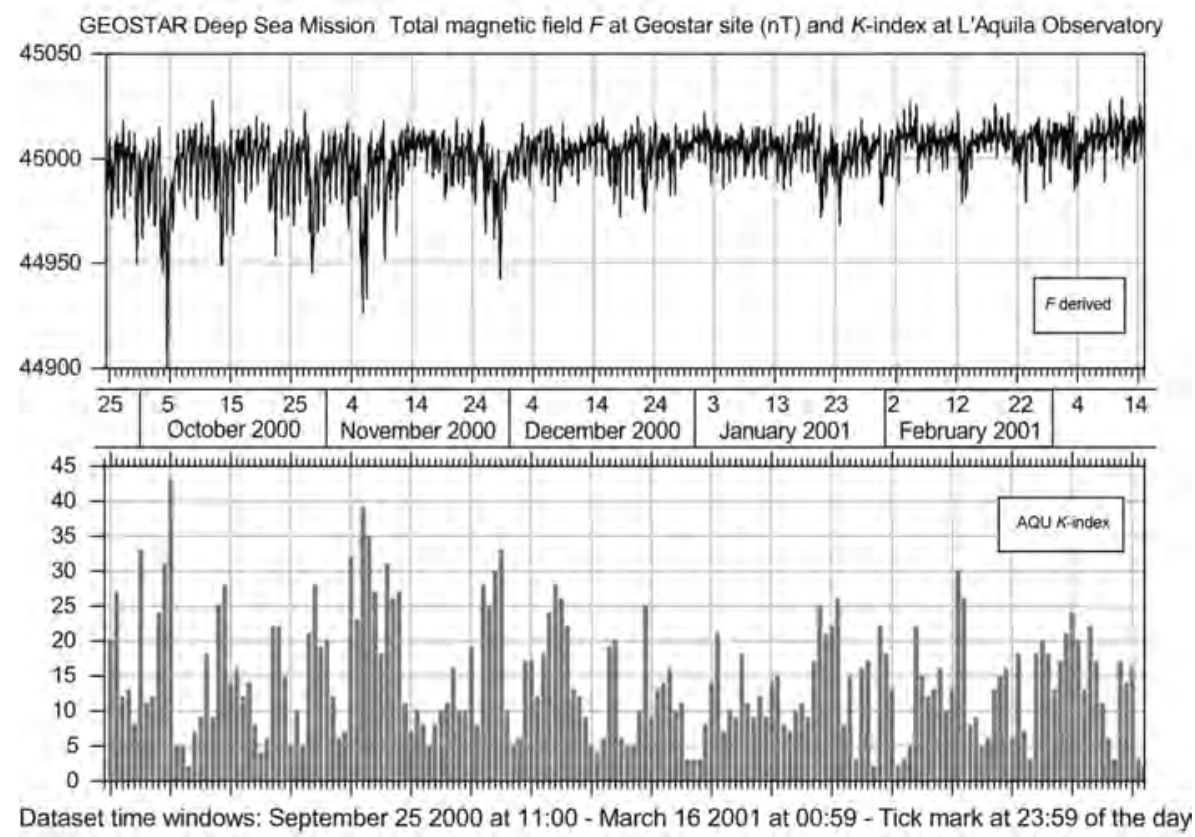

Fig. 11. Comparison between total magnetic $F$ and L'Aquila Observatory daily total $K$-index. 

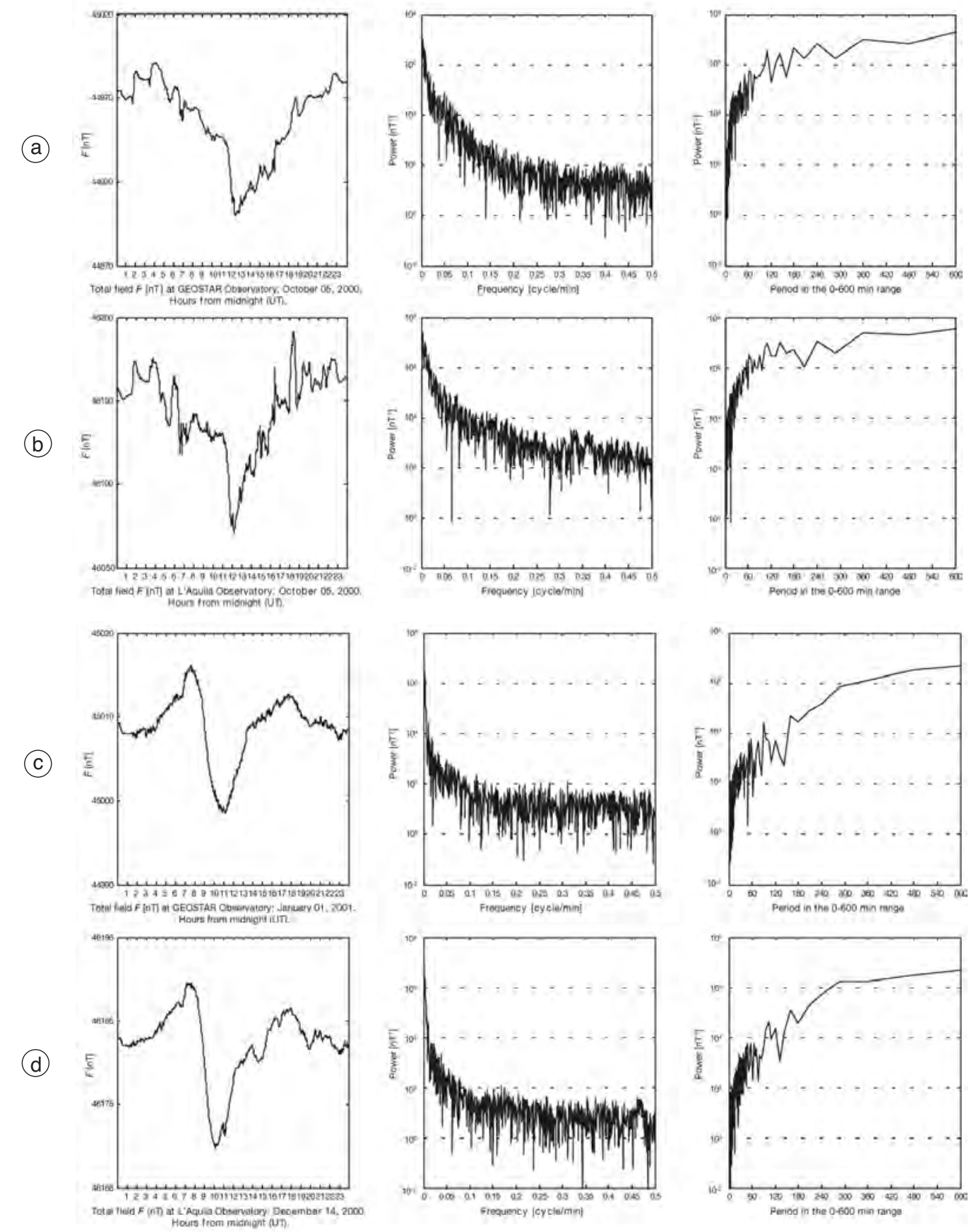

Fig. 12a-d. Spectral analysis of GEOSTAR (a, c) and L'Aquila (b, d) total intensity $F$ for October 5, 2000 (a, b) and January 01, 2001 (c, d). From left: temporal plot, spectrum with frequency and period at the abscissa. The corresponding daily total $K$-index was 43 (a, b) and 3 (c, d). 
(e)
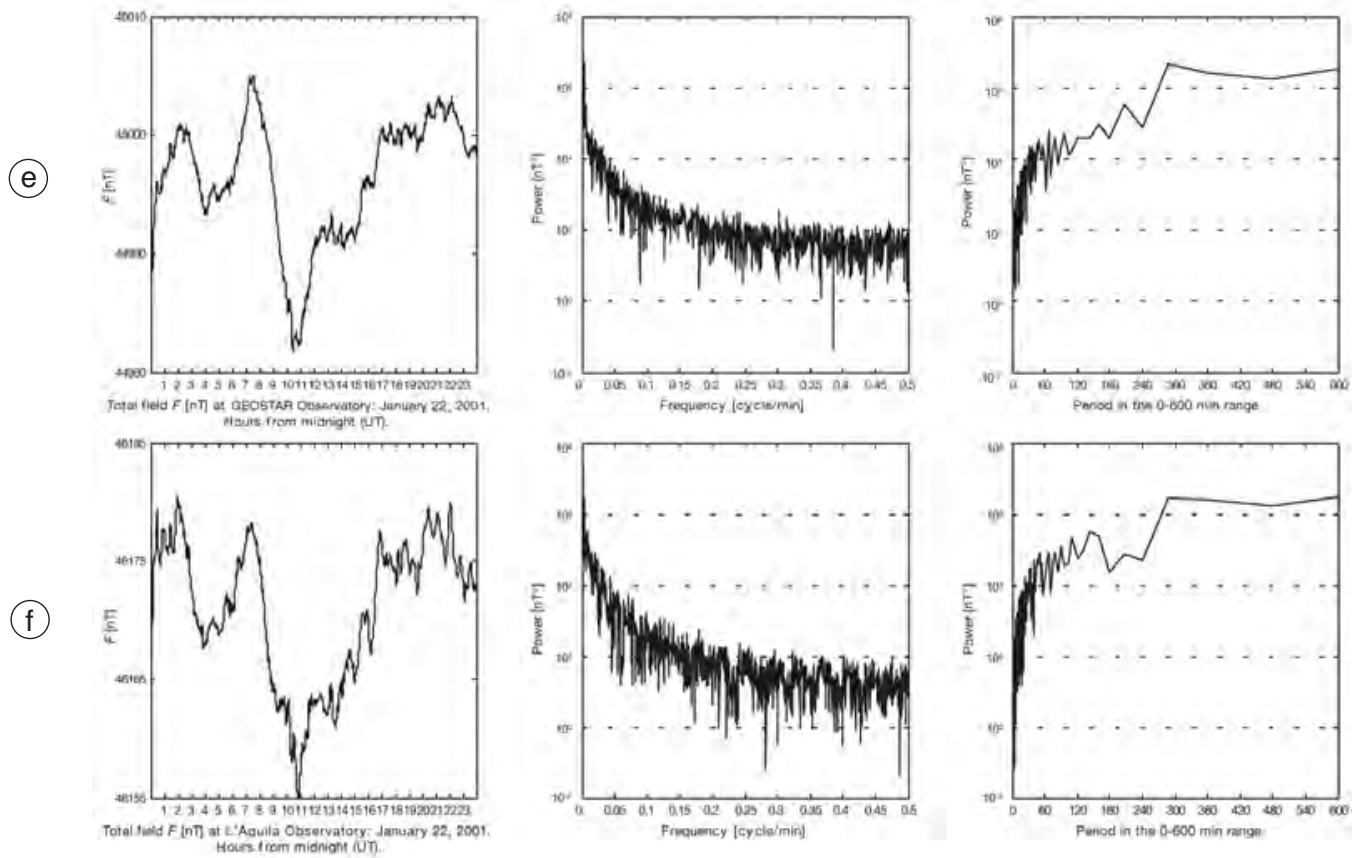

Fig. 12e,f. Spectral analysis of GEOSTAR (e) and L'Aquila (f) total intensity $F$ for January 22, 2001. From left: temporal plot, spectrum with frequency and period at the abscissa. The corresponding daily total $K$-index was 21 .

The selected days are October 5, 2000 (daily total $K$-index $=43$; strong magnetic activity), January 1, 2001 (daily total $K$-index $=3$; low magnetic activity), and January 22, 2001 (daily total $K$-index $=21$; moderate magnetic activity). The analysis of each daily segment of data (1440 data points) provides suitable spectral information over the frequency range from 0 to 0.5 cycle/min. The cases examined show in particular a difference between the L'Aquila and GEOSTAR power spectra, which becomes less and less evident with decreasing total $K$-index (fig. 12 a-f), i.e. with decreasing geomagnetic activity. This difference consists in a larger content of high frequency energy at L'Aquila than at the GEOSTAR site in the case of high geomagnetic activity, on the contrary the low frequency energy content remains of the same order of magnitude for any activity level. This behaviour can be explained by the screening effect of the seawater, especially on short period components and it is more marked when the total $K$-index has large values as in the case of October 5, 2000 when it was 43 . In this case the high frequency portion of the power spectrum of GEOSTAR data asymptotically approaches values that are at least two orders of magnitude lower than the analogous spectrum obtained for the signal simultaneously recorded at L'Aquila. On the contrary low frequency variations affect measurements taken at L'Aquila and those taken at the GEOSTAR site practically in the same way. The seawater screening effect is also confirmed in the following description of how the induction mechanism dominates at longer periods.

\section{Preliminary analysis of EM induction and electrical conductivity}

A combination of the vertical and horizontal components of the fluxgate magnetometer dataset allows the estimation of the so-called Parkinson arrows (or induction arrows) of the $\mathrm{Ge}$ - 
omagnetic Depth Sounding (GDS) technique (e.g., Armadillo et al., 2001). They provide both quantitative and qualitative indications of the presence of conductivity contrasts near the point of measurement from the complex ratios (i.e. the so-called transfer functions) of the Fourier transforms of the horizontal and the vertical magnetic components at different periods (the longer the periods the deeper the corresponding sounded layer). In the graphic representation, the real parts of such arrows are directed towards regions with higher conductivity. This technique can be successfully applied also in the band limited seafloor environment. We applied the GDS technique to the time variations of the GEOSTAR magnetic data for a preliminary selection of 12 events (duration: $512 \mathrm{~min}$ ) from 6 magnetically undisturbed days (not shown here). In general, most of the arrows become larger as the period increases. This confirms that at the GEOSTAR site (i.e. at a depth of $2000 \mathrm{~m}$ ) the bulk of electromagnetic induction generated by variations in the geomagnetic field contains mainly long period components. Moreover, most of the arrows point toward north or north-east, indicating that a higher electrical conductivity contrast is located along these directions with respect to Ustica Island. This anomaly could be possibly due to the asthenospheric upweeling of the Tyrrhenian Basin. We expect that this aspect will be better investigated by analysing the whole dataset.

\section{Conclusions}

The magnetic experiment of the GEOSTAR project succeeded in providing reliable magnetic data for most of the period of the deep water mission. Techniques were applied in order to calibrate and orientate the GEOSTAR magnetometers. Comparison with ground stations (Gibilmanna and Ustica sites) and observatories (L'Aquila and Castello Tesino) showed a good agreement, confirming the quality of the data.

Power spectral analysis was performed on selected data segments (characterized by the daily total $K$-index). The results showed that the energy in the power spectra at short periods is larger at L'Aquila than at the GEOSTAR site especially when the magnetic activity is high. On the contrary, the energy involved at longer periods is of the same order of magnitude at the GEOSTAR site and at L'Aquila for any level of geomagnetic activity.

Finally, induction studies showed that a higher electrical conductivity contrast is located north-north-east of the Ustica Island.

In the future, more detailed analyses will be needed to better evaluate the data, together with a careful comparison with the corresponding satellite data from the Oersted and Champ missions (e.g., Reigber et al., 2005).

\section{Acknowledgements}

The GEOSTAR project was funded by the EC under the Marine Science and Technology Programme (contract n. MAS3-CT95-0007).

We thank all the people who were involved directly or indirectly in the GEOSTAR project. Among them, Giuseppe («Pino» to his friends) Smriglio merits special mention for his great patience, strength and humanity expressed to everybody during this time. His premature departure has left a great emptiness in all of us.

We thank David Barraclough, Mioara Mandea and Antonio Meloni for providing important comments for improving the original version of the paper.

\section{REFERENCES}

Armadillo, E., E. Bozzo, V. Cerv, A. De Santis, D. Di Mauro, M. Gambetta, A. Meloni, J. Pek and F. SpeRANZA (1998): Geomagnetic depth sounding in the Northern Apennines (Italy), Earth Planets Space, 53, 385-396.

Beranzoli, L., A. De Santis, G. Etiope, P. Favali, F. FruGoni, G. Smriglio, F. Gasparoni and A. Marigo (1998): GEOSTAR: a Geophysical and Oceanographic Station for Abyssal Research, Phys. Earth Planet. Inter., 108 (2), 175-183.

Beranzoli, L., T. Braun, M. Calcara, D. Calore, R. Campaci, J.-M. Coudeville, A. De Santis, D. Di Mauro, G. Etiope, P. Favali, F. Frugoni, J.-L. Fuda, F. Gamberi, F. Gasparoni, H.W. Gerber, M. Marani, J. Marvaldi, C. Millot, P. Palangio, G. Romeo and G. SMriglio (2000): GEOSTAR: the first European long term seafloor observatory, Eos, Trans. Am. Geophys. Un., 81 (5), 45-49.

Beranzoli, L., T. Braun, M. Calcara, P. Casale, A. De 
Santis, G. D'Anna, D. Di Mauro, G. Etiope, P. FavaLi, J.-L. Fuda, F. Frugoni, F. Gamberi, M. Marani, C. Millot, C. Montuori and G. Smriglio (2003): Mission results from the first GEOSTAR Observatory (Adriatic Sea, 1998), Earth Planets Space, 55 (7), 361373.

Courtillot, V. and J.L. Le MouËL (1984): Geomagnetic secular variation impulses, Nature, 311, 709-716.

De Santis, A., D. Di Mauro, P. Favali, P. Palangio, G. Romeo and G. SMriglio (1999): GEOSTAR project: the performed seafloor mission in the Adriatic Sea, in Proceedings of MARELEC '99 Conference, Brest, France, 219-230.

Favali, P., G. Smriglio, L. Beranzoli, T. Braun, M. Calcara, G. D'Anna, A. De Santis, D. Di Mauro, G. Etiope, F. Frugoni, V. Iafolla, S. Monna, C. Montuori, S. Bozzoli, P. Palangio and G. Romeo (2002): Towards a permanent deep-sea observatory: the GEOSTAR European experiment, in Science-Technology Synergy for Research in Marine Environment: Challenges for the XXI Century, edited by L. BERANZOLI, P. Favali and G. SMriglio, Development in Marine Technology Series (Elsevier, Amsterdam), 12, 111-120.

FiLloux, J.H. (1987): Instrumentation and experimental methods for Oceanic Studies, in Geomagnetism, edited J. JaCOBS (Academic Press), vol. 1.

Gasparoni, F., D. Calore, R. Campaci and A. Marigo (1998): GEOSTAR - Development and test of an inno- vative benthic station for long-term observations at abyssal depths, in Proceedings of the IEEE Conference OCEANS'98 (on CD-ROM).

Gasparoni, F., D. Calore and R. Campaci (2002): From ABEL to GEOSTAR: development of the first European deep-sea scientific observatory, in Science-Technology Synergy for Research in Marine Environment: Challenges for the XXI Century, edited by L. BERANZOLI, P. FAVAli and G. SMriglio, Development in Marine Technology Series (Elsevier, Amsterdam), 12, 143-159.

Marvaldi, J., J. Blandin, Ch. Podeur, J.-M. Coudeville, J. Antoine, D. Barbot, D. Fellmann and D. Rhodes (1998): GEOSTAR - Development and test of a communication system for deep-sea benthic stations, in Proceedings of the IEEE Conference OCEANS '98 (on CD-ROM) .

Marvaldi, J., Y. Aoustin, G. Ayela, D. Batbot, J. Blandin, J.-M. Coudeville, D. Fellman, G. LoaËc, C. Podeur and A. Priou (2002): Design and realisation of communication systems for the GEOSTAR project, in Science-Technology Synergy for Research in Marine Environment: Challenges for the XXI Century, edited by L. Beranzoli, P. Favali and G. Smriglio, Development in Marine Technology Series (Elsevier, Amsterdam), 12, 161-181.

Reigber, C., H. Luhr, P. Scwintzer and J. Wickert (Editors) (2005): Earth Observation with CHAMP: Results from Three Years in Orbit (Springer, Berlin), pp. 628. 